McGill 95-42

August 1995

\title{
Hidden Finite Symmetries in String Theory and Duality of Dualities
}

\author{
Nemanja Kaloper \\ Department of Physics, McGill University \\ Montréal, Québec, Canada H3A 2T8 \\ email: kaloper@hep.physics.mcgill.ca
}

\begin{abstract}
Different compactifications of six-dimensional string theory on $M_{4} \times T^{2}$ are considered. Particular attention is given to the roles of the reduced modes as the $S$ and $T$ fields. It is shown that there is a discrete group of invariances of an equilateral triangle hidden in the model. This group is realized as the interchanges of the two-form fields present in the intermediate step of dimensional reduction in five dimensions. The key ingredient for the existence of this group is the presence of an additional $U(1)$ gauge field in five dimensions, arising as the dual of the Kalb-Ramond axion field strength. As a consequence, the theory contains more fourdimensional $S L(2, R)$ representations, with the resulting complex scalar axidilaton related to the components of the Kaluza-Klein vector fields of the naive dimensional reduction. An immediate byproduct of this relationship is a triadic correspondence among the fundamental string, the solitonic string, and a singular Brinkmann pp wave.
\end{abstract}

Submitted to Physics Letters $\mathbf{B}$ 
There has been much interest recently in studying strong-weak coupling duality symmetry and the relationship of the associated $S$ and $T$ fields in string theory in four dimensions [1]-[14]. These symmetries assist our taxonomy of different string constructions by providing links between them, predict the existence and properties of non-trivial topological configurations, and give means for constructing new configurations from the old ones. Generically, two kinds of dualities are encountered in string theory: 1$)$ the target space $(T)$ duality, which is realized as an $O(d, d)$ transformation group of moduli and gauge fields leaving the effective action invariant, and 2) the strong/weak coupling $(S)$ duality, which appears as an $S L(2, R)$ group of transformations of the complex scalar axidilaton field and gauge fields, that keep the equations of motion invariant but not the action in the naive dimensional reduction' Each group has a discrete subgroup believed to be an exact symmetry of string theory both in the string loop expansion and in the inverse string tension $\alpha^{\prime}$ expansion, with the difference that $T$ should be perturbatively exact in $\alpha^{\prime}$ and nonperturbatively exact in the string loop expansion, and vice-versa for $S$. It has been shown that in certain special cases these two symmetries can be interchanged, establishing a connection between either two different string theories (e.g. heterotic and type IIa, with specific compactifications from ten to four dimensions [6, 7, 9]), or between different compactifications within the same theory (e.g. regarding the duality of dualities map as a relationship between different vacua in the same string theory, which is allowed for special solutions with trivial fermions and Yang-Mills gauge fields) [4, 6, 7]. In this letter I will focus on the latter case, and assuming that the model considered is the heterotic string theory demonstrate how the results obtained so far can be extended to obtain another, alternative, compactification providing new representations for the dualities. These symmetries become manifest when the Kalb-Ramond axion field strength is replaced by its Hodge dual (weighted with the dilaton), which in five dimensions plays the role of an additional vector gauge field [10]. The resulting representations are related by a finite group of symmetries of the equilateral triangle $D_{4}$, realized by mixing the modulus with the dilaton and permuting the resulting $U(1)$ gauge fields in the intermediate step of dimensional reduction in five dimensions. One of these transformations is just the standard scale-factor $T$ duality in five dimensions, whereas another is precisely the duality of dualities transformation conjectured by Duff and Khuri [6], and derived in more general

\footnotetext{
${ }^{1}$ There exists a formulation of the theory where the manifest invariance of the action under $S L(2, R)$ transformations is restored, see [3].
} 
circumstances by Duff [7]. As the $D_{4}$ group has only two independent generators, we see that these two transformations are in fact sufficient to describe the full action of the group. The result of their combinations, however, is non-trivial because they lead to different compactifications to four dimensions, interchanging four dimensional moduli with the axidilaton, and concomitantly realizing a multiplicity of string dualities. I will finally discuss a straightforward application to the fundamental string solution in six dimensions, and using its dual relationship to the solitonic string solution and a Brinkmann pp wave, propose that there should be a string subtraction scheme in which the solitonic solution should be exact to all orders in the inverse string tension expansion.

In order to define the $T$ and $S$ symmetries, we need to look at the effective theory in four dimensions. Here only the basics of dimensional reduction for the bosonic sector of string theory will be presented (see e.g. [2] for a more complete account). Our starting point is the effective action describing the graviton multiplet in six dimensions, to the lowest order in $\alpha^{\prime}$ [2, 7, 9]:

$$
S=\int d^{6} x \sqrt{\bar{g}} e^{-\Phi}\left\{\bar{R}+(\bar{\nabla} \Phi)^{2}-\frac{1}{12} \bar{H}_{\mu \nu \lambda}^{2}\right\}
$$

The bar denotes six-dimensional quantities throughout the article. The fields $\Phi$ and $\bar{H}_{\mu \nu \lambda}=$ $\partial_{\mu} \bar{B}_{\nu \lambda}+$ cyclic permutations are the six dimensional dilaton and three-form axion fields respectively. The explicit representation of the string duality symmetries is accomplished by the Kaluza-Klein reduction of this theory to four dimensions, resulting in an action with additional scalar and gauge fields coupled to the graviton, axion and dilaton. The gauge fields arise from the cross-terms in the metric ("shift functions") and the two-form axion potential, and are invariant under the corresponding $U(1)$ symmetries. The scalars are the breathing modes of the compact directions and the two-form axion field components living in the compact submanifold. The duality symmetries are included in the model via the couplings of these additional fields. Thus, in terms of the six-dimensional background, the metric and matter fields are

$$
\begin{aligned}
d \bar{s}^{2}= & g_{\mu \nu} d x^{\mu} d x^{\nu}+G_{A B}\left(d y^{A}+V_{\mu}^{A} d x^{\mu}\right)\left(d y^{B}+V_{\nu}^{B} d x^{\nu}\right) \\
\bar{B}= & \frac{1}{2}\left(B_{\mu \nu}-\frac{1}{2}\left(V_{\mu}^{A} B_{\nu A}-V^{A}{ }_{\nu} B_{\mu A}\right)+B_{A B} V^{A}{ }_{\mu} V^{B}{ }_{\nu}\right) d x^{\mu} d x^{\nu} \\
& +\left(B_{\mu A}-B_{A B} V^{B}{ }_{\mu}\right) d x^{\mu} d y^{A}+\frac{1}{2} B_{A B} d y^{A} d y^{B} \\
\Phi= & \phi+\frac{1}{2} \ln \left|\operatorname{det}\left(G_{A B}\right)\right|
\end{aligned}
$$


The vector fields $V^{A}{ }_{\mu}, B_{\mu A}$ are the $U(1)$ gauge fields mentioned above which are coming from the cross-terms in the metric and the axion, respectively. Their field strengths will be denoted by $V_{\mu \nu}^{A}$ and $H_{\mu \nu A}$. The corrections in (2) proportional to $V$ are necessary to disentangle the resulting four-dimensional gauge symmetries.

After straightforward but tedious algebraic manipulations with the reduced action, using gauge-invariance as the guide and switching between the tangent-space and holomorphic bases, we can rewrite the action in the following form:

$$
S=\int d^{4} x \sqrt{g} e^{-\phi}\left\{R+(\nabla \phi)^{2}+\frac{1}{8} \operatorname{Tr}(\mathcal{L} \nabla \mathcal{M})^{2}-\frac{1}{4} \mathcal{F}_{\mu \nu}^{T} \mathcal{M} \mathcal{F}^{\mu \nu}-\frac{1}{12} H_{\mu \nu \lambda}^{2}\right\}
$$

The capital $T$ denotes matrix transposition. The $\sigma$-model fields $\mathcal{M}$ appear after rearranging the scalar moduli fields. The correspondence is given by

$$
\mathcal{M}=\left(\begin{array}{cc}
g^{-1} & -g^{-1} b \\
b g^{-1} & g-b^{T} g^{-1} b
\end{array}\right) \quad \mathcal{L}=\left(\begin{array}{cc}
0 & \mathbf{1} \\
\mathbf{1} & 0
\end{array}\right)
$$

where $g, b$ and $\mathbf{1}$ are $2 \times 2$ matrices defined by the dynamical degrees of freedom of the metric and the axion: $g=\left(G_{A B}\right)$ and $b=\left(B_{A B}\right)$. The vector multiplet is obtained from the Kaluza-Klein and axionic gauge fields as follows:

$$
\mathcal{A}_{\mu}=\left(\begin{array}{c}
B_{\mu A} \\
V_{\mu}^{A}
\end{array}\right) \quad \mathcal{F}_{\mu \nu}=\left(\begin{array}{c}
H_{\mu \nu A} \\
V_{\mu \nu}^{A}
\end{array}\right)
$$

and the axion field strength can be rewritten as

$$
H_{\mu \nu \lambda}=\partial_{\mu} B_{\nu \lambda}-\frac{1}{2} \mathcal{A}_{\mu}^{T} \mathcal{L F}_{\nu \lambda}+\text { cyclic permutations }
$$

Note that $\mathcal{M}^{T}=\mathcal{M}$ and $\mathcal{M}^{-1}=\mathcal{L} \mathcal{M} \mathcal{L}$. Thus we see that $\mathcal{M}$ is a symmetric element of $O(2,2, R)$. Therefore an $O(2,2, R)$ rotation $\mathcal{M} \rightarrow \Omega \mathcal{M} \Omega^{T}$ and $\mathcal{F} \rightarrow \Omega \mathcal{F}$, while changing $\mathcal{M}$ and $\mathcal{F}$, is a symmetry of the action and the equations of motion. The continuous $O(2,2, R)$ symmetry is believed to be broken down to $O(2,2, Z)$ by non-perturbative effects. This symmetry contains the $T$-duality symmetry of string theory [1, 2].

To see how the other duality symmetry, $S$, comes into play, we need to first conformally rescale the metric to the Einstein frame, which in four dimensions is given by $\hat{g}_{\mu \nu}=\exp (-\phi) g_{\mu \nu}$, and then replace the three-form axion field strength in four dimensions by its dual pseudoscalar field. The correspondence between them is

$$
H_{\mu \nu \lambda}=e^{2 \phi} \sqrt{\hat{g}} \epsilon_{\mu \nu \lambda \rho} \hat{\nabla}^{\rho} a
$$


The hat here denotes the Einstein conformal frame. If we introduce the complex axidilaton field $\Psi=a+i \exp (-\phi)$ and the self-dual and antiself-dual parts of the gauge fields $\hat{\mathcal{F}}_{\mu \nu}^{ \pm}=$ $\hat{\mathcal{F}}_{\mu \nu} \pm i \mathcal{L} \mathcal{M}^{*} \hat{\mathcal{F}}_{\mu \nu}$, where ${ }^{*} \hat{\mathcal{F}}_{\mu \nu}=\frac{1}{2} \sqrt{\hat{g}} \epsilon_{\mu \nu \lambda \rho} \hat{\mathcal{F}}^{\lambda \rho}$ is the Hodge dual of the two-form $\hat{\mathcal{F}}_{\mu \nu}$, we can rewrite the action (3) as

$$
S=\int d^{4} x \sqrt{\hat{g}}\left\{\hat{R}+\frac{2 \hat{\nabla}_{\mu} \Psi \hat{\nabla}^{\mu} \Psi^{\dagger}}{\left(\Psi-\Psi^{\dagger}\right)^{2}}+\frac{1}{8} \operatorname{Tr}(\mathcal{L} \hat{\nabla} \mathcal{M})^{2}+\frac{i}{16} \Psi \hat{\mathcal{F}}_{\mu \nu}^{-T} \mathcal{M} \hat{\mathcal{F}}^{-\mu \nu}-\frac{i}{16} \Psi^{\dagger} \hat{\mathcal{F}}_{\mu \nu}^{+T} \mathcal{M} \hat{\mathcal{F}}^{+\mu \nu}\right\}
$$

where $\dagger$ denotes complex conjugation. A careful examination of the equations of motion derived from this action shows that they are invariant under the following set of transformations:

$$
\Psi \rightarrow \Psi^{\prime}=\frac{\alpha \Psi+\beta}{\gamma \Psi+\delta} \quad \hat{\mathcal{F}}_{\mu \nu}^{-} \rightarrow \hat{\mathcal{F}}_{\mu \nu}^{\prime-}=(\gamma \Psi+\delta) \hat{\mathcal{F}}_{\mu \nu}^{-} \quad \alpha \delta-\beta \gamma=1
$$

where $\alpha, \beta, \gamma$ and $\delta$ are all real numbers. Also, $\hat{\mathcal{F}}_{\mu \nu}^{+}$transforms like the complex conjugate of $\hat{\mathcal{F}}_{\mu \nu}^{-}$. The equations of motion remain invariant under (9) because the gauge equations of motion (Euler-Lagrange and Bianchi) are interchanged and the axidilaton equation itself is invariant, as well as the gauge energy-momentum tensor (the term from which it is derived changes by a boundary term only). However, the action (8) itself is not invariant. This can be seen from the fact that (9) changes the sign of the gauge terms in the action. The transformations (9) combine to form the $\mathrm{SL}(2, \mathrm{R})$ group. This symmetry group is referred to as the strong/weak, or $S$, duality. Again, it is believed to be broken down to $S L(2, Z)$ by instanton effects; the relevant physical symmetry is thus this discrete group [1, 3].

The above illustrates the essential properties of the two duality symmetries. These two symmetries appear to be considerably different in that $T$ leaves the action invariant and $S$ does not. This problem is easily resolved with the introduction of several Lagrange multipliers, after which the action can be rewritten in a manifestly $O(2,2)$ and $S L(2)$ invariant form [3]. It is then reasonable to ask if the two groups can be related or perhaps interchanged. This "duality of dualities" has in fact been recently shown to hold between different string theories [7, 9], and the implications are still being studied. Essentially, it is based on the possibility to reduce the model after dualizing the three-form in six dimensions, which transforms the four-dimensional moduli fields, as will be outlined later.

A generalization of this duality of dualities can be attained in the following way. The dimensional descent can be taken more gradually. Choosing any one of the two cyclic coordinates, the starting six-dimensional action can be first reduced to five dimensions, again 
according to the standard Kaluza-Klein prescription. Because the axion moduli are absent, the formulas for the reduced modes are now simpler than in the four-dimensional case. The reduced axionic contributions and the dilaton are given by

$$
\begin{aligned}
d \bar{s}_{6}^{2} & =g_{5 \mu \nu} d x^{\mu} d x^{\nu}+\zeta e^{2 \sigma}\left(d y+V_{\mu} d x^{\mu}\right)^{2} \\
\bar{B} & =\frac{1}{2}\left(B_{5 \mu \nu}-\frac{1}{2}\left(V_{\mu} B_{\nu}-V_{\nu} B_{\mu}\right)\right) d x^{\mu} \wedge d x^{\nu}+B_{\mu} d x^{\mu} \wedge d y \\
\Phi & =\phi_{5}+\sigma
\end{aligned}
$$

For the sake of simplicity, we have dropped the superscript " $y$ " from the gauge fields $V^{y}{ }_{\mu}$ and $B_{\mu y}$, but have retained the subscript " 5 " on the metric, the dilaton, and the two-form axion to distinguish them from the four-dimensional quantities. In this equation $\zeta= \pm 1$ allows for the possibility that the reduced coordinate $y$ is either space- or time-like, respectively. (We leave this option open because the duality transformations can be used for generating solutions, and so are insensitive of the topological character of the Killing coordinates.) One must keep in mind that the gauge fields $V$ and $B$ in five dimensions are not identical to the gauge fields appearing in the direct $6 \rightarrow 4$ reduction. Rather, they contain different admixtures of the effective four-dimensional gauge fields. Obviously, since we could choose either of the two compact coordinates to integrate out first, there will be in general two different possibilities for the intermediate five-dimensional theory. The relationship between the five- and four-dimensional fields can be found by comparing (2) and (10), and will not be listed here. Then, the reduced three-form axion in five dimensions is

$$
H_{5 \mu \nu \lambda}=\partial_{\mu} B_{5 \nu \lambda}-\frac{1}{2} V_{\mu} H_{\nu \lambda}-\frac{1}{2} B_{\mu} V_{\nu \lambda}+\text { cyclic permutations }
$$

The reduced action then becomes, after dividing by $\int d y$,

$$
S=\int d^{5} x \sqrt{g_{5}} e^{-\phi_{5}}\left\{R_{5}+\left(\nabla \phi_{5}\right)^{2}-(\nabla \sigma)^{2}-\frac{\zeta}{4} e^{2 \sigma} V_{\mu \nu}^{2}-\frac{\zeta}{4} e^{-2 \sigma} H_{\mu \nu}^{2}-\frac{1}{12} H_{5 \mu \nu \lambda}^{2}\right\}
$$

At this point, we need to conformally transform the metric to the five-dimensional Einstein frame. This is necessary in order to bring the gauge-scalar couplings in the manifestly symmetric form, as we will show shortly. Namely, after we conformally transform to the Eisntein frame, and rescale the dilaton field $\phi_{5}$ to $\eta=\phi_{5} / \sqrt{3}$, we will find that the scalar fields $\vec{\sigma}=(\sigma, \eta)$ can be viewed as an $O(2)$ doublet. Moreover, the Einstein frame metric will be decoupled, and thus invariant under these isospin rotations. We will then show that the 
gauge kinetic terms couple to the scalar doublet via a set of Toda-like functions $\exp \left(\vec{Q}_{k} \cdot \vec{\sigma}\right)$ for some constant isovectors $\vec{Q}_{k}, k \in 1, \ldots, 3$, to be determined. These couplings can transform covariantly among themselves under discrete subgroups of $O(2)$, if the isovectors lie on vertices of regular polygons. Obviously, if these transformations are followed by permutations of the associated gauge fields, the induced representation may be a symmetry of the action. Thus, the conformal transformation is given by $\hat{g}_{5 \mu \nu}=\exp \left(-2 \phi_{5} / 3\right) g_{5 \mu \nu}$. The next step is to replace the Kalb-Ramond axion field strength by its Hodge dual two-form, which introduces the third gauge field in the model [10]. This dualization of the Kalb-Ramond field strength is carried out with the help of a Lagrange multiplier vector field $X_{\mu}$, to account properly for the axion Bianchi identity $\epsilon^{\sigma \rho \mu \nu \lambda} \partial_{\rho} H_{5 \mu \nu \lambda}=-\frac{3}{2} \epsilon^{\sigma \rho \mu \nu \lambda} V_{\rho \mu} H_{\nu \lambda}$. This vector field actually becomes the gauge potential for the gauge field $X_{\mu \nu}$. The relation between it and the Kalb-Ramond field strength is

$$
H_{5 \mu \nu \lambda}=\frac{1}{2} e^{4 \phi_{5} / 3} \sqrt{\hat{g}_{5}} \epsilon_{\sigma \rho \mu \nu \lambda} \hat{X}^{\sigma \rho}
$$

The full five-dimensional action in the Einstein frame can then be rewritten as

$$
\begin{aligned}
S=\int d^{5} x \sqrt{\hat{g}_{5}}\left\{\hat{R}_{5}\right. & -(\hat{\nabla} \eta)^{2}-(\hat{\nabla} \sigma)^{2}-\frac{\zeta}{4} e^{2(\sigma-\eta / \sqrt{3})} \hat{V}_{\mu \nu}^{2} \\
& \left.-\frac{\zeta}{4} e^{-2(\sigma+\eta / \sqrt{3})} \hat{H}_{\mu \nu}^{2}-\frac{\zeta}{4} e^{4 \eta / \sqrt{3}} \hat{X}_{\mu \nu}^{2}+\frac{1}{4} \frac{\epsilon^{\sigma \rho \mu \nu \lambda}}{\sqrt{\hat{g}_{5}}} X_{\sigma} V_{\rho \mu} H_{\nu \lambda}\right\}
\end{aligned}
$$

We note that the anomaly-like term, trilinear in the gauge fields, is symmetric under arbitrary permutations of these fields. To extend the permutation symmetry of the anomaly-like term, we note that in light of the aforementioned $O(2)$ isospace interpretation, the coupling vectors which determine the scalar-gauge couplings are given as $\vec{Q}_{1}=(2,-2 / \sqrt{3}), \vec{Q}_{2}=$ $(-2,-2 / \sqrt{3})$, and $\vec{Q}_{3}=(0,4 / \sqrt{3})$ and lie on the vertices of an equilateral triangle. Since the invariance transformations of the coupling triangle are in fact a discrete subgroup of $O(2)$, we see that they must be invariances of the action. Indeed, it is straightforward to verify that the transformations from the group $D_{4}$, of the form

$$
\left(\begin{array}{c}
\sigma \\
\frac{\phi_{5}}{\sqrt{3}}
\end{array}\right) \rightarrow\left(\begin{array}{c}
\sigma^{\prime} \\
\frac{\phi_{5}^{\prime}}{\sqrt{3}}
\end{array}\right)=\bar{\Omega}_{2}\left(\begin{array}{c}
\sigma \\
\frac{\phi_{5}}{\sqrt{3}}
\end{array}\right) \quad\left(\begin{array}{c}
V_{\mu} \\
B_{\mu} \\
X_{\mu}
\end{array}\right) \rightarrow\left(\begin{array}{c}
V_{\mu}^{\prime} \\
B_{\mu}^{\prime} \\
X_{\mu}^{\prime}
\end{array}\right)=\bar{\Omega}_{3}\left(\begin{array}{c}
V_{\mu} \\
B_{\mu} \\
X_{\mu}
\end{array}\right)
$$

where the matrices $\bar{\Omega}_{2}$ and $\bar{\Omega}_{3}$ belong to a two-dimensional and a three-dimensional representation of $D_{4}$, respectively, leave the form of the action unchanged. Under these transformations, the three different gauge fields of the five-dimensional action are interchanged 
together with the couplings. We note that because the five-dimensional Einstein frame metric does not change under (15), the world-sheet metric transforms according to $g_{5 \mu \nu} \rightarrow g_{5 \mu \nu}^{\prime}=$ $\exp \left(2\left(\phi_{5}^{\prime}-\phi_{5}\right) / 3\right) g_{5 \mu \nu}$.

One of these transformations is just the standard scale-factor $T$ duality in five dimensions, which interchanges $V$ and $B$, and inverts the $\sigma$ field: $V \leftrightarrow B$ and $\sigma \rightarrow-\sigma$. Another transformation, with $\sigma^{\prime}=\left(\sigma-\phi_{5}\right) / 2, \phi_{5}^{\prime}=-\left(3 \sigma+\phi_{5}\right) / 2$, and $B \leftrightarrow X$ is precisely the duality of dualities transformation derived by Duff [7]. This can be seen as follows. The original duality of dualities map was realized in six dimensions, starting with the action (1), and transforming the fields according to [7, 9]

$$
\begin{aligned}
\bar{g}_{\mu \nu} \rightarrow \bar{g}_{\mu \nu}^{\prime} & =e^{-\Phi} \bar{g}_{\mu \nu} \\
\bar{H}_{\mu \nu \lambda} \rightarrow \bar{H}_{\mu \nu \lambda}^{\prime} & =\frac{1}{6} e^{-\Phi} \sqrt{\bar{g}} \epsilon_{\mu \nu \lambda \alpha \beta \gamma} \bar{H}^{\alpha \beta \gamma} \\
\Phi \rightarrow \Phi^{\prime} & =-\Phi
\end{aligned}
$$

The action in terms of the transformed fields is:

$$
S=\int d^{6} x \sqrt{\bar{g}^{\prime}} e^{-\bar{\Phi}^{\prime}}\left\{\bar{R}^{\prime}+\left(\nabla \bar{\Phi}^{\prime}\right)^{2}-\frac{1}{12} \bar{H}_{\mu \nu \lambda}^{\prime 2}\right\}
$$

i.e., it is form-invariant. If we now attempt to represent these formulas in five dimensions, using (10), we immediately obtain these transformation rules for the reduced fields:

$$
\begin{aligned}
2 \sigma^{\prime} & =\sigma-\phi_{5} \\
2 \phi_{5}^{\prime} & =-\phi_{5}-3 \sigma \\
g_{5 \mu \nu}^{\prime} & =e^{2\left(\phi_{5}^{\prime}-\phi_{5}\right) / 3} g_{5 \mu \nu} \\
V_{\mu}^{\prime} & =V_{\mu}
\end{aligned}
$$

which we recognize as a part of the sought correspondence. The last step is to find how the reduced axion fields transform. In order to do it, we first have to separate between the reduced two- and three-forms. Noting from (10) that $\bar{H}_{\mu \nu y}=H_{\mu \nu}$, and also recalling the definition of the reduced two-form axion potential (10), we find that

$$
\begin{aligned}
H_{\mu \nu}^{\prime} & =-\frac{\zeta}{6} e^{-\phi_{5}} \sqrt{g_{5}} \epsilon_{\mu \nu \alpha \beta \gamma} H_{5}^{\alpha \beta \gamma} \\
\bar{H}_{\mu \nu \lambda}^{\prime} & =\frac{1}{2} e^{-\phi_{5}} \sqrt{g_{5}} \epsilon_{\mu \nu \lambda \alpha \beta}\left(e^{-2 \sigma} H^{\alpha \beta}-\zeta V_{\gamma} H_{5}^{\alpha \beta \gamma}\right)
\end{aligned}
$$


Note that (19) are given in terms of the string-frame metric. It is obvious that RHS of the first of these equations is just the inverse of the Eq. (13). This gives $H_{\mu \nu}^{\prime}=X_{\mu \nu}$. Also, in the second equation, $\zeta$ does not multiply the first term in the parenthesis because it appeared through the square $\zeta^{2}=1$. Now, we must recall that $\bar{H}_{\mu \nu \lambda}$ is not the gauge invariant quantity in five dimensions. It transforms anomalously under Kaluza-Klein gauge transformations. The second term in the second equation of (19) accounts for this. We rectify this problem by replacing it with the gauge-invariant field strength, which we find by combining (10) and (11):

$$
H_{5 \mu \nu \lambda}=\bar{H}_{\mu \nu \lambda}-\left(V_{\mu} H_{\nu \lambda}+\text { cyclic permutations }\right)
$$

This is the form which we need to dualize in order to obtain the third gauge field in five dimensions. Switching to the Einstein frame and introducing the Hodge dual of the gaugeinvariant five-dimensional three-form $X_{\mu \nu}^{\prime}$ we get the relation between it and $\bar{H}_{\mu \nu \lambda}^{\prime}$ :

$$
\bar{H}_{\mu \nu \lambda}^{\prime}=\frac{1}{2} e^{4 \phi_{5} / 3} \sqrt{\hat{g}_{5}} \epsilon_{\mu \nu \lambda \alpha \beta} \hat{X}^{\prime \alpha \beta}-\left(V_{\mu} H_{\nu \lambda}^{\prime}+\text { cyclic permutations }\right)
$$

Equating the RHS of this equation with the RHS of the second equation of (19), substituting in $H_{\mu \nu}^{\prime}=X_{\mu \nu}$ and recalling again the definition of the dual gauge field $X$ (13), we finally obtain

$$
X_{\mu \nu}^{\prime}=H_{\mu \nu} \quad H_{\mu \nu}^{\prime}=X_{\mu \nu}
$$

i.e. exactly the permutation rule we have discussed before. Thus we see that the sixdimensional string/string duality map reduces to one of the $D_{4}$ transformations.

The two transformations discussed above are sufficient to describe the full action of the group $D_{4}$, because it has only two independent generators. The result of their combinations, however, is non-trivial because in combination with the arbitrariness of the direction taken to reduce the action from six to five, and further to four dimensions, they lead to different compactifications to four dimensions, interchanging four-dimensional real moduli with the complex axidilaton, which results in exchanges between the $S$ and $T$ duality symmetries. In the approach presented here, after applying one of these transformations, one only needs to replace the new $X$ field with its dual three-form and complete the reduction of the model to four dimensions, reading off the relevant degrees of freedom.

As an interesting consequence of this symmetry there appears a triadic relationship among the fundamental string, the solitonic string and a singular Brinkmann pp wave in six dimensions. As these solutions all possess the same number of supersymmetries in six dimensions, 
in the theory in which they are embedded, perhaps this triangular relationship should not come as too much of a surprise. Let us now outline how the transformations we have discussed above establish a connection between these solutions. The fundamental string solution is 15

$$
\begin{aligned}
d \bar{s}^{2} & =\left(1+\frac{C}{r^{2}}\right)^{-1}\left(-d t^{2}+d y^{2}\right)+d r^{2}+r^{2} d l_{3} \\
\bar{B} & =\frac{C}{C+r^{2}} d y \wedge d t \\
e^{-\Phi} & =1+\frac{C}{r^{2}}
\end{aligned}
$$

with $d l_{3}$ the line element of the unit three-sphere $S^{3}$. It represents the target space configuration of an elementary string state in six dimensions, and is singular at $r=0$. The solitonic string solution can be obtained from (23) by applying the duality map defined by the equation (16), or equivalently, by reducing on $y$, applying the triangle transformation $\sigma^{\prime}=\left(\sigma-\phi_{5}\right) / 2, \phi_{5}^{\prime}=-\left(3 \sigma+\phi_{5}\right) / 2, B \leftrightarrow X$, and then lifting the new configuration back to six dimensions. The result is [6, 7, 8]

$$
\begin{aligned}
d \bar{s}^{2} & =-d t^{2}+d y^{2}+\left(1+\frac{C}{r^{2}}\right) d r^{2}+\left(C+r^{2}\right) d l_{3} \\
\bar{H} & =-2 C \omega_{3} \\
e^{-\Phi} & =\left(1+\frac{C}{r^{2}}\right)^{-1}
\end{aligned}
$$

The $\omega_{3}$ is the volume form of the unit three-sphere $S^{3}$. This solution is actually nonsingular as $r \rightarrow \infty$, as can be seen by changing coordinates to $\rho=\ln r$, and realizing that $\rho \rightarrow-\infty$ is geodesically excluded from the manifold. This, and the fact that the string axion charge is carried not by matter sources but by the topology of the target space, is the reason why the solution is called solitonic.

In the above two solutions, the string charge was carried by the axion field. As we have seen above, from the triangular symmetry there still exists the possibility to trade the axionic charge for the Kaluza-Klein one. Applying the five-dimensional scale factor duality $\sigma \rightarrow-\sigma$, $V \leftrightarrow B$ to the fundamental string (23), we find

$$
d \bar{s}^{2}=-\left(1+\frac{C}{r^{2}}\right)^{-1} d t^{2}+\left(1+\frac{C}{r^{2}}\right)\left(d y-\frac{C}{C+r^{2}} d t\right)^{2}+d r^{2}+r^{2} d l_{3} \quad \bar{B}=0 \quad \Phi=0
$$


Note that the matter fields are trivial. Changing the coordinates to $u=t-y, v=t+y$, we can rewrite the solution as

$$
d \bar{s}^{2}=d u d v+\frac{C}{r^{2}} d u^{2}+d r^{2}+r^{2} d l_{3} \quad \bar{B}=0 \quad \Phi=0
$$

which we immediately recognize as a Brinkmann pp wave singular at $r=0$ [16, 17, 18]. Strictly speaking, this metric has an undesirable property that if $y$ is compactified periodically there appear closed null curves, manifest after the transformation to null coordinates. Also, from the string point of view, this solution is infinitely degenerate, because we can absorb $C$ away by a coordinate transformation $d u \rightarrow d u^{\prime}=d u \sqrt{C}, d v \rightarrow d v^{\prime}=d v / \sqrt{C}$. It is then not difficult to see that the last $D_{4}$ inversion, characterized by $\sigma^{\prime}=\left(\sigma+\phi_{5}\right) / 2$, $\phi_{5}^{\prime}=-\left(3 \sigma-\phi_{5}\right) / 2, V \leftrightarrow X$ merely interchanges the solitonic (24) and the pp wave (26) solutions. Thus there are no other non-trivial $D_{4}$ images of the fundamental string (23).

There are two immediate observations based on the triangular correspondence of these string solutions. First, we know that both the fundamental string and the pp wave are exact solutions to all orders in $\alpha^{\prime}$. This comes about because both solutions have a conserved chiral current on the world-sheet (being an $F$-type (fundamental) and a $K$-type conformal field theory (pp wave) discussed recently by Horowitz and Tseytlin [17]). This establishes that the $T$-duality relationship between the pp wave and the fundamental string is in fact exact, modulo field redefinitions. Namely, the string subtraction schemes in which these two solutions are exact may differ by finite renormalizations (i.e., string field redefinitions), implying that the $T$ duality could also pick up these corrections. Given this and the triangular relationship of these solutions with the solitonic string, it is then compelling to conjecture that there should exist a scheme in which the solitonic string is also exact to all orders in $\alpha^{\prime}$. This would be necessary to establish the equivalence of all the $D_{4}$ transformations. An argument regarding the exactness of the solitonic string was put forth earlier, but it required the introduction of a Yang-Mills gauge field to cancel $\alpha^{\prime}$ corrections [6]. In light of the triangular relationship, however, this may not be necessary. A related observation is that since both the fundamental and solitonic strings are identified as the states in string spectrum, for $D_{4}$ to be an exact symmetry of string theory the pp wave must also belong to this spectrum. Since the wave is infinitely degenerate, and it has $S^{3}$ target-space symmetry it would be interesting to see if it can be related to a five-dimensional variant of the recently conjectured massless black holes associated with conifold singularities [14]. 
Note added. Upon the completion of the research presented here, I have received the paper entitled "Four Dimensional String/String/String Triality" by M.J. Duff, J.T. Liu and J. Rahmfeld, preprint \# CTP-TAMU-27/95/hep-th/9508094, [19] where the triality relationship among the heterotic, type $I I_{A}$ and type $I I_{B}$ string theory was established, and which overlaps in some length with the present work. Also, a related analysis is presented in the paper "U-Dualtiy and Integral Structures" by P.S. Aspinwall and D.R. Morrison, preprint \# CLNS-95/1334/hep-th/9505025, [20], since published in Phys. Lett. B355 141.

\section{Acknowledgements}

The author would like to thank R.R. Khuri and R.C. Myers for stimulating conversation and helpful comments on the manuscript. This work was supported in part by NSERC of Canada, and in part by an NSERC postdoctoral fellowship.

\section{References}

[1] A. Sen, Phys. Lett. B271 (1991) 295; Phys. Lett. B274 (1992) 34; Nucl. Phys. B404 (1993) 109; Phys. Rev. Lett. 69 (1992) 1006; S.F. Hassan and A. Sen, Nucl. Phys. B375 (1992) 103; Nucl. Phys. B405 (1993) 143.

[2] J. Maharana and J.H. Schwarz, Nucl. Phys. B390 (1993) 3-32.

[3] J.H. Schwarz and A. Sen, Phys. Lett. B312 (1993) 105-114; Nucl. Phys. B411 (1994) 35-63.

[4] P. Binetruy, Phys. Lett. B315 (1993) 80-87.

[5] A. Giveon, M. Porrati and E. Rabinovici, Phys. Rep. 244 (1994) 244.

[6] M.J. Duff and R.R. Khuri, Nucl. Phys. B411 (1994) 473-486.

[7] M.J. Duff, Nucl. Phys. B442 (1995) 47-63. 
[8] M.J. Duff and R. Minasian, Nucl. Phys. B436 (1995) 47-63; M.J. Duff, R.R. Khuri and J.X. Lu, Physics Reports, 259 (1995) 507-528. M.J. Duff, S. Ferrara, R.R. Khuri and J. Rahmfeld, preprint hep-th/9506057, in press in Phys. Lett. B356 (1995) 479.

[9] A. Sen, Nucl. Phys. B440 (1995) 421-440; preprint TIFR-TH-95-16/hep-th/9504027.

[10] E. Witten, Nucl. Phys. B443 (1995) 85-126.

[11] C. Vafa and E. Witten, preprint HUTP-95-A015/hep-th/9505053; A. Sen and C. Vafa, preprint HUTP-95-A028/ hep-th/9508064.

[12] E. Bergshoeff, R. Kallosh and T. Ortin, Phys. Rev. D51 (1995) 3009-3016.

[13] C.M. Hull, preprint QMW-TH-95-25/hep-th/9506194; E. Bergshoeff, C.M. Hull and T. Ortin, preprint UG-3-95/hep-th/9503022; C.M. Hull and P.K. Townsend, Nucl. Phys. B438 (1995) 109-137.

[14] A. Strominger, preprint hep-th/9504090.

[15] A. Dabholkar, G. Gibbons, J.A. Harvey and F. Ruiz Ruiz, Nucl. Phys. B340 (1990) 33-55.

[16] A.A. Tseytlin, Nucl. Phys. B390 (1993) 153-172.

[17] G.T. Horowitz and A.A. Tseytlin, Phys. Rev. D51 (1995) 2896-2917; Phys. Rev. 73 (1994) 3351-3354; Phys. Rev. D50 (1994) 5204-5224.

[18] E. Bergshoeff, R. Kallosh and T. Ortin, Phys. Rev. D50 (1994) 5188-5192; R. Kallosh, preprint SU-ITP-94-18/hep-th/9406093.

[19] M.J. Duff, J.T. Liu and R. Minasian, preprint CTP-TAMU-26-95/hep-th/9506126.

[20] P.S. Aspinwall and D.R. Morrison, preprint hep-th/9505025, and published in Phys. Lett. B355 (1995) 141. 